(1)

CrossMark

\section{Arterial hypertension and the risk of severity and mortality of COVID-19}

To the Editor:

We read with great interest the recently published study by GuAN et al. [1]. To the best of our knowledge this is the first large-scale study that focuses on independent clinical risk factors associated with a composite outcome (death, use of a ventilator, or intensive care unit (ICU) requirement), using a Cox regression model.

The study found that arterial hypertension was the most prevalent comorbidity (16.9\%), followed by diabetes (8.2\%), cardiovascular disease (3.7\%), cerebrovascular disease (1.9\%), COPD (1.5\%) and malignancy (1.1\%). In the Cox regression model, after adjustment for age and smoking status, the independent risk factors associated with the composite outcome were malignancy (hazard ratio (HR) 3.5, 95\% CI 1.60-7.64), COPD (HR 2.68, 95\% CI 1.42-5.05), diabetes (HR 1.59, 95\% CI 1.03-2.45) and hypertension (HR 1.58, 95\% CI 1.07-2.32).

We have some concerns about the results of that analysis, especially those related to arterial hypertension. Initial Chinese epidemiological studies suggested that cardiovascular diseases (including arterial hypertension) and diabetes were associated with mortality or severity in patients affected by coronavirus disease 2019 (COVID-19). Wu and MCGoogan [2] stated that in 44672 patients with confirmed COVID-19, the overall case fatality rate was $2.3 \%$, and that this was elevated in comorbid conditions: $10.5 \%$ for cardiovascular disease, $7.3 \%$ for diabetes, $6.3 \%$ for chronic respiratory disease and $6 \%$ for hypertension. GuAN et al. [3] found that in 1099 patients with confirmed COVID-19, hypertension was a more prevalent condition in those who lead the primary composite end-point (admission to ICU, the use of mechanical ventilation or death; $35.8 \%$ versus $13.7 \%)$ and in those with severe disease (23.7\% versus 13.4\%). RUAN et al. [4] also described that in 150 patients with confirmed COVID-19, cardiovascular disease and hypertension were more frequent in cases who died compared with those who were discharged ( $19 \%$ versus $0 \%, \mathrm{p}<0.001$, and $43 \%$ versus $28 \%, \mathrm{p}=0.07$, respectively). Moreover, a systematic review and meta-analysis that included 46248 infected patients found that the most prevalent comorbidity was hypertension (mean \pm SD $17 \pm 7 \%$; 95\% CI 14-22\%) and that, compared with non-severe patients, the pooled odds ratios of hypertension and cardiovascular disease were OR 2.36 (95\% CI 1.46-3.83) and OR 3.42 (95\% CI 1.88-6.22), respectively [5]. All the evidence seems to be concordant. Remarkably, none of these studies performed a multivariable adjustment. The effect of arterial hypertension on the severity or mortality outcome could be explained by potential confounders.

To address this unsolved issue, we conducted a medical literature search in PubMed on 8 April 2020, using the following strategy:

\section{(COVID-19[tiab] OR SARS-CoV-2[tiab]) AND (mortality[tiab] OR severity[tiab])}

We included in the analysis the papers that were designed to find clinical predictors of mortality or severity for severe acute respiratory syndrome coronavirus 2 (SARS-CoV-2) infection. The data form these articles is shown in table 1.

Both studies shown in table 1 found that, like hypertension, age and history of coronary artery disease were predictors of COVID-19 severity or mortality in the univariate analysis. But in both studies, hypertension was not included in the final multivariate logistic regression model. The first study included the following in the final multivariate model to predict critical COVID-19: elevated troponin I (TnI)

@ERSpublications

Arterial hypertension implies the risk of a hard COVID-19 outcome. This may be explained by potential confounding factors, such as age and cardiovascular disease. https://bit.ly/2Kr6Ax4

Cite this article as: Leiva Sisnieguez CE, Espeche WG, Salazar MR, et al. Arterial hypertension and the risk of severity and mortality of COVID-19. Eur Respir J 2020; 55: 2001148 [https://doi.org/10.1183/ 13993003.01148-2020]. 
TABLE 1 Studies that evaluated arterial hypertension and its association with severity or mortality in coronavirus disease 2019 using a multivariate logistic regression model

\begin{tabular}{|c|c|c|c|c|c|c|c|c|}
\hline \multirow{3}{*}{$\begin{array}{l}\text { First } \\
\text { author } \\
\text { [ref.] }\end{array}$} & \multirow{3}{*}{$\begin{array}{c}\text { Subjects } \\
n\end{array}$} & \multicolumn{3}{|c|}{ Comparison groups } & \multicolumn{4}{|c|}{ Outcome } \\
\hline & & \multirow[t]{2}{*}{ Critical } & \multirow[t]{2}{*}{ Non-critical } & \multirow[t]{2}{*}{ p-value } & \multicolumn{4}{|c|}{ Critical disease status } \\
\hline & & & & & OR $(95 \% \mathrm{CI})$ & p-value & OR $(95 \% \mathrm{CI})$ & p-value \\
\hline \multirow{3}{*}{ Chen [6] } & & Did not survive & Survived & $p$-value & \multicolumn{4}{|c|}{ Death } \\
\hline & & & & & \multicolumn{2}{|c|}{ Univariate analysis } & \multicolumn{2}{|c|}{ Multivariate analysis } \\
\hline & & & & & OR $(95 \% \mathrm{CI})$ & p-value & OR $(95 \% \mathrm{CI})$ & p-value \\
\hline Zнои [7] & 191 & $48 \%$ & $23 \%$ & $<0.001$ & $3.05(1.57-5.92)$ & 0.001 & $\begin{array}{l}\text { Authors only } \\
\text { significant pr }\end{array}$ & $\begin{array}{l}\text { orted } \\
\text { ctors }\end{array}$ \\
\hline
\end{tabular}

Data are presented as OR (95\% CI), unless otherwise stated. " : the outcome for CHEN [6] was the critical disease status, the outcome for ZHou [7] was death.

(OR 26.91, 95\% CI 4.09-177.23; p=0.001) and history of coronary artery disease (OR 16.61, 95\% CI 2.29120.58; $\mathrm{p}=0.005$ ) [6]. The multivariable regression performed to predict COVID-19 death in the second study [7] included age (OR 1.10, 95\% CI 1.03-1.17; $\mathrm{p}=0.0043$ ), a higher sequential organ failure assessment score (OR 5.65, 95\% CI 2.61-12.23; $\mathrm{p}<0.001$ ) and D-dimer $>1 \mu \mathrm{g} \cdot \mathrm{mL}^{-1}$ on admission (OR $18.42,95 \%$ CI 2.64-128.55; $\mathrm{p}=0.003)$.

As previously mentioned, prevalent cardiovascular disease is associated with higher mortality and severity of COVID-19 in the univariate analysis $[2,4,5]$. Thus, the association is also maintained in at least one of the multivariate models discussed, but not between hypertension and hard COVID-19 outcomes. In this sense, it is well known that: 1) the prevalence of essential hypertension and coronary artery disease increases with age; and 2) coronary disease and hypertension frequently coexist in the same patient. In this way, the association between hypertension and COVID-19 mortality or severity could be partly explained by the increased age and higher prevalence of cardiovascular disease. Both are well-known risk factors for mortality in critical patients. Furthermore, it has been suggested that patients with cardiac injury (elevated TnI) have a worse prognosis, suggesting specific target organ damage by SARS-CoV-2 [8]. This finding could explain why patients with prevalent cardiovascular disease are associated with worse COVID-19 outcomes.

For these reasons, we suggest that in order to conclude that hypertension could be an independent predictor of COVID-19 mortality or severity, the model should be adjusted for cardiovascular disease, to exclude its potential confounding effect.

Carlos Enrique Leiva Sisnieguez @ , Walter Gastón Espeche and Martín Rogelio Salazar

Faculty of Medical Sciences, National University of La Plata, Buenos Aires, Argentina, and Argentinian Society of Arterial Hypertension (SAHA).

Correspondence: Carlos Enrique Leiva Sisnieguez, National University of La Plata, Medical Sciences Faculty, 71 y 115 Pabellón Académico, La Plata, Buenos Aires 1900, Argentina. E-mail: cleiva@med.unlp.edu.ar

Received: 12 April 2020 | Accepted: 15 April 2020

Conflict of interest: None declared.

\section{References}

1 Guan W-J, Liang W-H, Zhao Y, et al. Comorbidity and its impact on 1590 patients with COVID-19 in China: a nationwide analysis. Eur Respir J 2020, 55: 2000547.

$2 \mathrm{Wu} \mathrm{Z}$, McGoogan JM. Characteristics of and important lessons from the coronavirus disease 2019 (COVID-19) outbreak in China: summary of a report of 72314 cases from the Chinese Center for Disease Control and Prevention. JAMA 2020; 13: 1239-1242.

3 Guan WJ, Ni ZY, Hu Y, et al. Clinical characteristics of coronavirus disease 2019 in China. N Engl J Med 2020; 382: $1708-1720$.

4 Ruan Q, Yang K, Wang W, et al. Clinical predictors of mortality due to COVID-19 based on an analysis of data of 150 patients from Wuhan, China. Intensive Care Med 2020; 46: 846-848. 
5 Yang J, Zheng Y, Gou X, et al. Prevalence of comorbidities and its effects in coronavirus disease 2019 patients: a systematic review and meta-analysis. Int J Infect Dis 2020; 94: 91-95.

6 Chen C, Chen C, Yan JT, et al. Analysis of myocardial injury in patients with COVID-19 and association between concomitant cardiovascular diseases and severity of COVID-19. Zhonghua Xin Xue Guan Bing Za Zhi 2020; 48: E008.

7 Zhou F, Yu T, Du R, et al. Clinical course and risk factors for mortality of adult inpatients with COVID-19 in Wuhan, China: a retrospective cohort study. Lancet 2020; 395: 1054-1062.

8 Shi S, Qin M, Shen B, et al. Association of cardiac injury with mortality in hospitalized patients with COVID-19 in Wuhan, China. JAMA Cardiol 2020; in press [https://doi.org/10.1001/jamacardio.2020.0950].

\section{Cardiovascular comorbidity and its impact on patients with COVID-19}

From the authors:

We truly appreciate the comments from C.E. Leiva Sisnieguez and colleagues, who have performed a further analysis on the potential association between cardiovascular comorbidities and the clinical outcomes of coronavirus disease 2019 (COVID-19), particularly the mortality). We also applaud the suggestion to thoroughly adjust for potential confounding factors when interpreting the association between specific categories of cardiovascular comorbidities (e.g. hypertension) and the clinical outcomes of COVID-19. To this end, we have attempted to incorporate the cardiovascular diseases (including coronary heart disease) into the multivariate regression model [1]. Findings of the model indicated a prominent collinearity between hypertension and coronary heart disease, and we have therefore elected to retain hypertension in the regression model for further analyses.

Like other comorbidities, such as COPD, information about cardiovascular comorbidities was derived from patient self-report, which does not preclude under-reporting. Therefore, the percentage of patients with cardiovascular diseases might have been underestimated given the urgency of data collection (history taking) within the wards during the outbreak. Our findings could also have been attributed to the relatively low proportion of patients with coexisting hypertension and coronary heart disease in our study. Nonetheless, the overall proportion of patients with comorbidities in our study [1] was in keeping with previous publications [2-6]. Our findings were likely to be generalisable to other populations worldwide.

The cause of the association between cardiovascular diseases and the poor clinical outcome of COVID-19 may be multifaceted, including, but not limited to, age and cardiac dysfunction caused by viral infections. As ours was a cross-sectional case study, causality could not be inferred from the study design. Dynamic monitoring of cardiovascular symptoms, cardiac function and laboratory markers might help unravel the underlying pathways linking cardiovascular diseases to the poor clinical outcomes of COVID-19.

@ERSpublications

Comorbid hypertension correlates with poorer outcomes in patients with COVID-19 https://bit.ly/ 2zoT9f0

Cite this article as: Guan W-jie, Liang W-hua, He J-xing, et al. Cardiovascular comorbidity and its impact on patients with COVID-19. Eur Respir J 2020; 55: 2001227 [https://doi.org/10.1183/13993003.012272020].

Wei-jie Guan ${ }^{1,3}$, Wen-hua Liang ${ }^{2,3}$, Jian-xing $\mathrm{He}^{2}$ and Nan-shan Zhong ${ }^{1}$

${ }^{1}$ State Key Laboratory of Respiratory Disease and National Clinical Research Center for Respiratory Disease, the First Affiliated Hospital of Guangzhou Medical University, Guangzhou Medical University, Guangzhou, China. ${ }^{2}$ Dept of Thoracic Oncology and Surgery, China State Key Laboratory of Respiratory Disease and National Clinical Research Center for Respiratory Disease, the First Affiliated Hospital of Guangzhou Medical University, Guangzhou, China.

${ }^{3}$ Wei-jie Guan and Wen-hua Liang are joint first authors.

Correspondence: Jian-xing He, Dept of Thoracic Surgery, the First Affiliated Hospital of Guangzhou Medical University; China State Key Laboratory of Respiratory Disease and National Clinical Research Center for Respiratory Disease, Guangzhou, China. E-mail: drjianxing.he@gmail.com 
Received: 17 April 2020 | Accepted: 17 April 2020

Author contributions: Wei-jie Guan and Wen-hua Liang drafted the manuscript; all authors provided critical review of the manuscript and approved the final draft for publication.

Conflict of interest: Wei-jie Guan has nothing to disclose. Wen-hua Liang has nothing to disclose. Jian-xing He has nothing to disclose. Nan-shan Zhong reports grants from National Health Commission and Dept of Science and Technology of Guangdong Province, during the conduct of the study.

Support statement: Supported by National Health Commission, Dept of Science and Technology of Guangdong Province. The funder had no role in the conduct of the study. Funding information for this article has been deposited with the Crossref Funder Registry.

\section{References}

1 Guan W-j, Liang W-h, Zhao Y, et al. Comorbidity and its impact on 1590 patients with COVID-19 in China: a nationwide analysis. Eur Respir J 2020; 55: 2000547.

2 Placzek HED, Madoff LC. Association of age and comorbidity on 2009 influenza A pandemic H1N1-related intensive care unit stay in Massachusetts. Am J Public Health 2014; 104: e118-e125.

3 Booth CM, Matukas LM, Tomlinson GA, et al. Clinical features and short-term outcomes of 144 patients with SARS in the greater Toronto area. JAMA 2003; 289: 2801-2809.

4 Alqahtani FY, Aleanizy FS, Ali Hadi Mohammed R, et al. Prevalence of comorbidities in cases of Middle East respiratory syndrome coronavirus: a retrospective study. Epidemiol Infect 2018; 5: 1-5.

5 Badawi A, Ryoo SG. Prevalence of comorbidities in the Middle East respiratory syndrome coronavirus (MERS-CoV). Int J Infect Dis 2016; 49: 129-133.

6 Alanazi KH, Abedi GR, Midgley CM, et al. Diabetes mellitus, hypertension, and death among 32 patients with MERS-CoV infection, Saudi Arabia. Emerging Infect Dis 2020; 26: 166-168. 